\title{
Investigar: una experiencia para cuestionar-nos
}

Artículo de reflexión

Research: An Experience to Question Ourselves Pesquisar: uma experiência para nos questionar

\author{
Mónica Rocío Barón Montaño* \\ Luz Stella Cañón Cueca**
}

Para citar este artículo:

Barón, M. y Cañón, L. (2018). Investigar: una experiencia para cuestionar-nos. Pedagogía y Saberes, 49, 115-125.

* Profesora Secretaría de Educación de Bogotá. Estudiante de Doctorado en Educación de la Universidad do Estado do Rio de Janeiro-UERJ e investigadora de grupo Diferença, desigualdade e educação escolar da juventude-CNPq.

Correo electrónico: mkron@hotmail.com

Código ORCID: http://orcid.org/0000-9991-7026-4464

** Profesora Secretaría de Educación de Bogotá. Doctora en Educación e investigadora del Grupo Historia de la Educación. Correo electrónico: stellaboreal@yahoo.com

Código ORCID: http://orcid.org/0000-0003-0159-8262 


\title{
Resumen
}

Este artículo de reflexión en torno a la experiencia "Hacia un aprendizaje comprensivo de la investigación”, como estrategia que integra las dinámicas en el Colegio Pablo Neruda, presenta algunos abordajes sobre el papel que juega la investigación en el contexto escolar. Las autoras incitan a (re)pensar, en primer lugar el tema de la investigación en el ámbito escolar a partir del diálogo con autores como Larrosa y Kohan — quienes invitan a superar la visión tradicional de la investigación, configurándola como experiencia de vida—, Giroux — quien reconoce a los docentes como investigadores e intelectuales de la educacióny Masschelein —que propone rasgos de la investigación en educación como procesos para descifrar el mundo y su cotidianidad-; en segundo lugar, el rol social de los docentes y su vinculación con los procesos investigativos a partir de su quehacer pedagógico, desde una mirada orientada por autores como Aljure, Betancur y Suárez. Finalmente, tratan el tema de la investigación y su relación con los procesos escolares.

\section{Palabras clave}

investigación en educación; experiencia; ámbito escolar; rol del profesor

\begin{abstract}
This article is a reflection on the experience, "Towards a comprehensive learning of research," as a strategy that integrates the dynamics in the Pablo Neruda School and presents some approaches to the role of research in the school context. The authors encourage to (re)think, first the research in the school based on a dialogue with authors like Larrosa and Kohan-who encourage us to overcome the traditional view of research, configuring it as a life experience, - Girouxwho recognizes teachers as researchers and intellectuals of education- and Masschelein - what presents features of research in education as processes to decipher the world and its everyday life-; second the social role of teachers and their relationship with research processes from their pedagogical work, from an approach guided by authors such as Aljure, Betancur and Suárez. Finally, they talk about research and its relationship with school processes.
\end{abstract}

\section{Keywords}

research in education; experience; school environment; teacher's role

\section{Resumo}

Este artigo de reflexão em torno da experiência "Rumo a uma aprendizagem compreensiva da pesquisa”, como estratégia que integra as dinâmicas no Colégio Pablo Neruda, apresenta algumas abordagens sobre o papel da pesquisa no contexto escolar. As autoras incentivam (re)pensar, por uma parte, o tema da investigação no cenário escolar a partir do diálogo com autores como Larrosa e Kohan - que nos convidam a superar a visão tradicional da pesquisa, configurando-a como uma experiência de vida-, Giroux, que reconhece professores como pesquisadores e intelectuais da educação—y Masschelein — que apresenta características da pesquisa em educação como processos para decifrar o mundo e sua cotidianidade-; por outra parte, o papel social dos professores e sua relação com os processos de pesquisa a partir de seu trabalho pedagógico, em uma abordagem norteada por autores como Aljure, Betancur e Suárez. Finalmente, elas referem o tema da pesquisa e sua relação com os processos escolares.

\section{Palavras-chave}

pesquisa em educação; experiência; âmbito escolar; papel do professor 
No hay enseñanza sin investigación ni investigación sin enseñanza. Estos quehaceres se encuen-

tran cada uno en el cuerpo del otro. Mientras enseño, continúo buscando, indagando. Enseño porque busco, porque indagué, porque indago y me indago.

FREIRE, 2006, p. 30

\section{Un ejercicio de pensar la experiencia en investigación y pensar la investigación como una experiencia}

Considerando que el tema de la investigación ha venido permeando los currículos de algunas instituciones educativas en el ámbito de la educación básica y media, en el Colegio Pablo Neruda se ha optado por integrar las competencias investigativas al aula de clase. Esta iniciativa da cuenta de un interés por incluir otras formas de comprender el proceso de enseñanza y aprendizaje, evidenciando que, tal como lo afirma Roso (2010), "actualmente son innumerables las instituciones educativas que potencian e incentivan la investigación científica entre los estudiantes de Educación Secundaria, Bachillerato y Formación Profesional" (p. 101).

Ahora bien, ¿qué se entiende por investigación en el Colegio Pablo Neruda? ¿Qué significa en el contexto de esa institución investigar? ¿Es necesaria la relación e interacción entre enseñanza e investigación? ¿Es posible reivindicar la investigación en educación como experiencia? ¿Qué hace difícil integrar investigación y prácticas educativas? Sin duda alguna, cualquier juicio u opinión alrededor de estas grandes cuestiones induce a configurar diversas redes de significados, bien sea en el ámbito de la didáctica, el currículo o desde cualquier otro campo inserto en la práctica educativa, que circunscribe un universo de problematizaciones que incitan a (re)pensar el tema de la investigación en el ámbito escolar.

El acento interrogativo de estas enunciaciones indica, además, la necesidad de fortalecer un camino de afirmar -o desafirmar-, aprender -o desaprender- sobre aspectos que, por el hecho de no tener una respuesta directa y única, se presentan aquí como problemáticos, es decir, son inquietudes que se encuentran actualmente, y tal vez inagotablemente, en debate. Se trata entonces de querer transformar estas preguntas en "nuestras" preguntas, dudas que me colocan y nos colocan a prueba, las cuales, a la vez, se deben tornar "públicas", para que lo vivido a partir de la experiencia "Hacia un aprendizaje comprensivo de la investigación" en el Colegio Pablo Neruda ${ }^{1}$, no sobre la investigación, sino en la investigación, no sobre la experiencia, sino desde la experiencia, nos lleve a explorar en nosotros y en nuestras vivencias cómo habitamos las prácticas educativas y qué sentido tiene aquello que vivimos, considerando que, tal como afirma Larrosa y Kohan (2014), “[...] la experiencia, y no la verdad, es lo que da sentido a la educación" (p. 5).

En el marco de esta experiencia, que se ha cimentado durante 17 años, se reconoce un proceso que surge de una construcción colectiva en la que han participado diferentes actores educativos, y en cuyo trabajo se busca, a partir de la integración de los fundamentos de las pedagogías críticas, abordar aspectos de la realidad social. En este ejercicio académico, docentes y estudiantes han fortalecido su experiencia como investigadores, han desarrollado producción académica y participan en la transformación del contexto educativo a través del análisis de la realidad en la cual se encuentran inmersos.

En este sentido, la investigación en educación pretende ser configurada más allá de la tradición que la tipifica como un acceso a la verdad, basado y orientado en la "producción del conocimiento", ampliamente debatida en el marco de la llamada sociedad del conocimiento, en la que el carácter pedagógico de la investigación está determinado en todos los sentidos por aspectos cognitivos (tanto formales como substanciales). Lo que se pretende a partir del trabajo desarrollado es inspirarnos en nuestra propia experiencia para (re)pensar la investigación más allá de la tradición que la presenta como un ejercicio que "[...] trata las cuestiones y problemas relativos a la naturaleza, epistemología, metodología, fines y objetivos en el marco de la búsqueda progresiva de conocimiento en el ámbito educativo" (Arnal, Del Rincón y La Torre, 1994).

Dicho de otro modo, se trata de focalizar nuestra mirada en la investigación desde el lenguaje de la experiencia, una perspectiva que nos permita comprender lo que vivimos, lo que nos conmueve y motiva, tornando nuestros acontecimientos en caminos posibles para la investigación y configurándola, de este modo, como inspiradora de otros pensamientos y no solamente como un procedimiento o un esquema de actuación que genera por sí mismo sus propios

1 Este texto es el resultado de algunas reflexiones tejidas en el marco del trabajo que se ha venido desarrollando en el Colegio Pablo Neruda, institución de carácter público de Bogotá, que en sus dinámicas ha incorporado la experiencia pedagógica conocida como "Hacia un aprendizaje comprensivo de la investigación”, estrategia que busca plantear nuevos paradigmas para la formación de estudiantes con grandes competencias en y para la investigación. 
resultados. Es decir, se apunta a la investigación pensada como experiencia de vida, en palabras de Kohan (2015), "experiencias sobre la educación, sobre el nomadismo y la errancia, sobre una forma de investigación educativa que transforma, sobre unas relaciones pedagógicas y una forma de hacer escuela que conmueve e invita a emprender un viaje" (p.15)

A partir de esas hipótesis de pensamiento, hubo una interlocución con diferentes actores del Colegio Pablo Neruda, como docentes, estudiantes y egresados, quienes, a partir de cuestionamientos surgidos de las mismas prácticas y evidenciados en un diálogo enmarcado en el contexto de entrevistas semiestructuradas, expusieron sus puntos de vista frente a la importancia y la trascendencia del trabajo investigativo desarrollado por la institución. Dicha información fue analizada y contrastada teóricamente con autores como Jorge Larrosa, Walter Kohan y Jan Masschelein, quienes brindaron herramientas desde el punto de vista del trabajo pedagógico como experiencia y la relación de esta con el trabajo investigativo.

Estos abordajes fueron complementados con las teorizaciones existentes respecto al reconocimiento de los docentes como investigadores, para lo cual Henry Giroux presenta una perspectiva que los considera intelectuales importantes para el desarrollo de la sociedad, mirada que ha sido tradicionalmente cuestionada tanto desde las dimensiones de su formación como desde el mismo papel de la educación. Para este autor, es necesario que el rol del docente se consolide no solamente desde el punto de vista de su labor, sino a partir de un ejercicio de (re)pensarse como profesional reflexivo tanto de su práctica como de sus relaciones sociales, es decir, desde su propia experiencia.

Autores como el belga Jan Masschelein (2014) auxilian en este análisis al referir tres rasgos característicos de la investigación en educación considerados en este contexto: 1) una investigación es educativa porque pone en cuestión, en primer lugar, al propio investigador (su pensamiento y los límites de su pensamiento); 2) una investigación es educativa porque está dirigida no simplemente a dar cuenta de las realidades educativas, sino a suscitar la cuestión pedagógica que hay en ellas; 3) por último, una investigación educativa trata de tornar algo público, es decir, trata de descifrar al mundo en su cotidianidad y en su verdad, para propiciar una percepción sobre esa realidad que no había sido concebida (al menos de ese modo).

Probablemente, al ser abordada así, la investigación reivindica la práctica educativa como una experiencia, es decir, como algo que nos incita a problematizar nuestras formas de ver, decir y pensar "lo educativo", colocándonos en un camino para vernos, definirnos y pensarnos de otro modo, de manera que otras formas de ver, decir y pensar nos permitan (re)encontrar esa realidad vibrante que permea las prácticas y los discursos que conocemos como investigación educativa. Estos aspectos son representados por uno de los docentes de la institución de la siguiente manera:

Profesor 1: participar de la experiencia conocida como "Hacia un aprendizaje comprensivo de la investigación" ha sido muy impactante, no solo en mi vida laboral, sino que me he involucrado tanto, que es un tema que ha trascendido a mi vida personal, ¿sabe? De hecho, decidí continuar con mis estudios de posgrado a partir de muchas dudas que fueron construyéndose con mis estudiantes a medida que desarrollábamos los trabajos que tuve la oportunidad de orientar. Creo que es un trabajo que va más allá del simple papel del profesor en su rol de investigador, creo que, en mi caso, ha logrado trastocar, ¿cómo dicen? Mmmmm... mis fibras más íntimas (risas), me ha hecho poner en cuestión mi trabajo como docente, le debo mucho a esta experiencia, y lo reitero, no solo en lo que se refiere a la parte académica y laboral.

Es claro que pensar la investigación en el Colegio Pablo Neruda no es solo ponderar sobre cómo integrarla al conocimiento o al diseño curricular, implica de cierto modo exponernos, es decir, ser receptivos, abiertos, sensibles y dejarnos llevar por la curiosidad. Siguiendo El último Foucault (Abraham, 2011), la curiosidad que aquí referimos tiene que ver con el cuidado de sí y con la atención, en otras palabras, una curiosidad que implica una actitud de cuidado o de atención frente a nuestra realidad educativa y el mundo que configura sus cotidianos. Se trata de una mirada aguda y centrada en la realidad, en lo que nos acontece hoy, en el presente, y se trata también de una disponibilidad de no tomar como hecho ya preestablecido lo que somos y lo que hacemos.

Desde lo anterior, la investigación en el ámbito educativo se hace posible cuando tanto docentes como estudiantes indagan y reflexionan sobre su vida cotidiana y los problemas que surgen en ella, construyendo a partir de un trabajo dialógico otras posibilidades de saber. Esta forma de abordaje "responde a la manera como los maestros inciden en la transformación de la cultura escolar, introduciendo cambios en las prácticas tradicionales, y mejorando la calidad y las formas de aprender en las escuelas" (Gutiérrez, 2015, p. 9).

Con el fin de esclarecer lo que hasta este punto hemos esbozado, tal vez sea conveniente, a partir de los tres rasgos característicos de la investigación en 
educación presentados, ampliar algunas miradas que permitan aproximarnos a la idea de "investigación educativa" aquí mostrada, integrando, además, las propias narrativas de otros actores del Colegio Pablo Neruda, con las cuales será posible hacer una lectura de la investigación educativa.

\section{Investigación en educación: eso que me pone en cuestión}

No podemos desconocer que la propia idea de educación está ligada a la experiencia, lo que nos lleva a configurarla como un ejercicio que es vivido como algo que da mucho que pensar a sus protagonistas (estudiantes y profesores), aspecto que es referido por una de las estudiantes de último grado de nuestro colegio:

Estudiante 1: los profesores hacen un trabajo previo para que nosotros podamos identificar las cosas o temas que nos llaman la atención, eso que nos gusta y que nos genera intriga, curiosidad. Por ejemplo, yo estoy integrada a un grupo que se llama Narrativas y en ese grupo podemos analizar, pensar, mirar las cosas que tenemos nosotros, lo que hemos vivido, cómo las podemos compartir, cómo podemos contarlas de una forma que no sea tan plana y lineal, que sea interesante y que integre diferentes vivencias $\mathrm{y}$, por qué no, que integre hasta nuestros propios sentimientos y cómo eso también se relaciona hasta con lo que aquí en el colegio aprendemos.

Lo real y lo cotidiano de nuestros estudiantes comienza a configurar el currículo en nuestro colegio, considerando que no podemos desconocer que la experiencia pone en marcha el proceso de pensamiento; tal como lo afirma Arendt $(2005,2016)$, "Yo no creo que sea posible ningún curso de pensamiento sin experiencia personal. Todo pensar es un repensar, un repensar las cosas [...]" (p. 37). Y es en ese ejercicio de pensar y (re)pensar la experiencia con los otros y con nosotros mismos en el ámbito escolar en el que lo que conocemos como realidad adquiere valor, presencia y fuerza.

En el mismo ejercicio de pensar con nuestros estudiantes sobre la experiencia "Hacia un aprendizaje comprensivo de la investigación", otra alumna comentó:

Estudiante 2: [...] en la línea de educación financiera, que es a la cual pertenezco, también integramos historias reales, por ejemplo, con los profesores en nuestro grupo entrevistamos a varias personas del barrio y ellos nos contaban por qué muchas veces, aunque no querían, tenían que acudir al uso de las tarjetas de crédito, simplemente porque no tenían cómo cubrir sus gastos, eso eran unas historias hasta bien dramáticas, después de uno escucharlos, pensaba, yo ni había llegado a verlo de esa manera. Como que ahí uno se da cuenta que no es solamente tener una tarjeta con un crédito bueno, sino que lo más importante es cómo uno aprovecha eso y no deja que el tema se le convierta en un dolor de cabeza.

Anécdotas como estas permiten pensar nuestro trabajo pedagógico desde la perspectiva de la experiencia, poniéndonos en interacción, además, con las prácticas y las relaciones que confluyen en el quehacer pedagógico, en cuyo escenario se expone un saber con unos actores y cualidades particulares, el cual no siempre está establecido por las reglas del conocimiento objetivamente crítico o por las reglas de intencionalidad técnica o práctica, pero que es imprescindible como saber pedagógico.

En ese proceso, el docente del colegio como investigador no es únicamente un intelectual, proveedor o acumulador de conocimiento, sino que, para él, el significado formativo o educativo de la investigación trasciende el hecho de enriquecerse en conocimientos, así como el estudiante no es el receptor que se limita a apropiarse de la construcción de un conocimiento, reconocido como válido y verdadero. Pensar la investigación como experiencia en este contexto irrumpe en esa tradición y nos lleva a ponderar un cambio frente a nuestra existencia como docentes investigadores, tal como lo afirma un docente del colegio:

Profesor 2: yo he llegado a trabajar cosas con los estudiantes que en mi vida había pensado trabajar. Últimamente he pensado que, incluso mis ideas, mis palabras, mis propias representaciones de lo que es el trabajo en el aula han cambiado con relación a lo que ahora hago con los estudiantes, es como romper con el esquema de que solo lo que llevo preparado a la clase es lo que cuenta, que los temas que están predefinidos son los que les interesan a los muchachos, no, ellos traen unas ideas (risas), ellos quieren todo, ellos están en una época que parecen una esponjita, ellos quieren todo, uno les propone cosas, y ellos se motivan y empiezan a proponer y proponer.

Vale la pena referir aquí lo expresado por Larrosa (2009), quien afirma: "De ahí que el sujeto de la experiencia no sea el sujeto del saber, o el sujeto del poder, o el sujeto del querer, sino el sujeto de la formación y de la transformación" (p. 17); el docente, entonces, como sujeto también de la investigación, está siempre en apertura, se deja afectar por lo que acontece en su cotidianidad, haciendo de esta experiencia parte de su propio aprendizaje. 


\section{Investigación en educación: eso que me pasa}

Uno de los aspectos que en muchas ocasiones resultan más silenciados en los espacios institucionales, en donde se tejen procesos de investigación que buscan construcción y producción de saberes, y en los tiempos y dinámicas programadas para dicha construcción, es precisamente el de la experiencia, pero no cualquiera, sino la entendida como "eso que me pasa", en palabras de Larrosa (2009, p. 14).

Continuando con la secuencia propuesta en el análisis aquí abordado, retomamos el segundo rasgo de la investigación en educación presentado en párrafos anteriores y que hace referencia a la característica que le permite no solo dar cuenta de las realidades educativas, sino suscitar la cuestión pedagógica que hay en ellas. Aproximarnos a un abordaje que dé cuenta de este aspecto nos remite a una inquietud: ¿cómo integrar nuestra realidad para que consigamos problematizar de modo pedagógico nuestra forma de ver, decir y pensar lo educativo?

En este punto vale la pena referir la dimensión conocida como experiencia, señalando algunas de sus posibilidades e interrelaciones que conlleven a una lectura del encuentro educativo como una experiencia, es decir, a hacer de la investigación en educación la posibilidad de una experiencia.

La experiencia, en este escenario, no es otra cosa que nuestra propia relación con el mundo, con los otros y con nosotros mismos, a partir de la cual podemos afirmar que algo nos pasa, algo nos sucede. Entonces, el deseo de problematizar nuestras formas de ver, decir y pensar lo educativo está ligado a la experiencia, en el sentido que "[...] lo real solo ocurre si es experimentado: lo real es lo que nos pasa, lo que nos sucede en la experiencia" (Larrosa, 2009, p. 14). Por lo tanto, la experiencia es ese modo de relación con el mundo, con los otros y con nosotros mismos, en donde aquello que llamamos realidad adquiere sentido, presencia y fuerza.

Concebir la experiencia como aquello que brinda posibilidades para investigar en educación es arriesgarse, en primer lugar, a pensar lo educativo desde lo real, desde lo que vivimos, desde lo que se vive, pero, sobre todo, es aproximarnos a lo que personas concretas viven y experimentan en sí mismas. Al indagar con nuestros estudiantes sobre las dinámicas desarrolladas en el contexto del proyecto "Hacia un aprendizaje comprensivo de la investigación", se destacan intervenciones como:

Estudiante 3: [...] yo diría que lo principal es que lo que se hace [en el marco del proyecto], independiente de la línea a la que perteneces, lo que se hace es que se parte de un problema o una situación real, no es nada que se saca de un libro, son situaciones que se viven a diario ya sea por nosotros mismos o por nuestros vecinos, familiares, amigos. Solo que después se va especificando como lo que uno quiere trabajar y la forma como lo va a hacer, si es por la parte financiera o más por la parte de narrativas, por la parte artística, por la parte de historia, eso depende del gusto o interés de cada uno.

La cuestión que aquí citamos es que lo real solo acontece en la medida en que traspasamos el esquema de lo que ya sabemos, pensamos y conocemos, para que, a partir de una actitud de curiosidad y atención frente a nuestra vivencia educativa, sea posible integrar lo real en nuestros saberes y prácticas.

Lo real no puede ocurrir entonces como algo impuesto o predeterminado que busca solamente nuevos conocimientos (procesos en los que, muchas veces, ni docentes ni estudiantes son transformados, sino que solamente trastocan sus saberes y, eventualmente, sus habilidades) y en donde la experiencia "[...] está confinada en una jaula, en cuyo interior da vueltas y vueltas sobre sí misma, y de la que cada vez es más difícil hacerla salir" (Breton, 2009, p. 394).

Habría que cuestionarnos también si solo (re) conocemos lo real mientras esté medido, encuadrado, enjaulado por las acciones de institucionalización, tematización, caracterización, jerarquización, etc., que constituyen las lógicas propias de nuestros saberes y prácticas. Vale la pena referir aquí la narración de un docente del colegio que da cuenta de la actitud de algunos actores, en ocasiones renuentes, que se niegan la posibilidad de percibir esa realidad:

Profesor 3: es que mire, docentes como, por ejemplo, un profesor de matemáticas que no voy a nombrar aquí, un profesor de esos que le dice que no a todo, y ya en el segundo año [de puesta en marcha el proyecto], es decir, que en el primer año que fue como empezar la iniciativa, él no se integró, él no quería participar y efectivamente en ese primer año, no participó. Ya cuando él vio que toda la dinámica del colegio, que todos los profesores estaban en un proyecto, él se integró a uno, que fue un grupo de historia. Un grupo que hizo también un proyecto muy bonito sobre la cuestión de historia y sociedad y veían, digamos, la historia de las casas aquí en Fontibón y el tema aquí de las construcciones. Es que uno tenía de donde escoger, todos los proyectos eran muy bonitos. Entonces como este profe que le comento ya se sintió como por fuera de todo, y él era solo decir no porque no, pues se integró y después que se integró se dio cuenta de que "oiga, me estoy perdiendo de algo muy interesante” y ahí 
cambió, y si viera, comenzó a participar muchísimo, muchísimo y de hecho, es el momento que ya llevamos más de cinco años desde que se hizo esa experiencia y el profesor todavía sigue hablando con los estudiantes en ese sentido, porque él era uno que matemáticas es matemáticas y acá no se habla de nada más, y no, ahora uno lo escucha que integra sus historias de vida, él tiene en ese sentido, digamos que ... cambió su dinámica.

Situarse ante la investigación en educación como experiencia significa entonces es centrarse en las cualidades de lo que se vive, en hechos que corresponden a un espacio/tiempo, que se viven temporalmente, que están localizados en momentos y en lugares determinados, que están también atravesados por múltiples relaciones, y que, según quién los vive, reconfiguran diversos sentidos.

\section{Investigación en educación: eso que no había pensado}

El tercer y último rasgo que contribuye a este breve análisis refiere a la posibilidad que brinda la investigación en educación de tornar algo público, es decir, de tratar de descifrar el mundo en su cotidianidad y en su verdad, para conferir una percepción sobre esa realidad que no había sido considerada (al menos de ese modo). Es una posibilidad en la que lo cotidiano, convertido en foco de estudio, es hecho público, puesto o dispuesto en un escenario público, o, como diría Arendt (2016, p. 230), colocado al interior de una esfera pública: "[...] el término 'público' significa el propio mundo, en cuanto es común a todos nosotros y diferenciado de nuestro lugar poseído privadamente en él".

Pensar la investigación como experiencia para descifrar el mundo implica configurarnos como "[...] territorios de paso, como una superficie de sensibilidad en la que algo pasa y en la que 'eso que me pasa' al pasar por mí, deja una huella, una marca, un rastro, una herida" (Larrosa, 2009, p. 25).

Comprendemos que todo lo que sucede actualmente en el mundo nos es totalmente accesible; podría decirse que esto obedece a la escenificación de nuevas formas de transmisión de la información y la comunicación, pero más allá de estos recursos, se trata también de una dinámica social en la que confluyen nuevas redes, comunidades y medios de comunicación social.

Hoy vemos cómo la oferta ilimitada de información y conocimiento está a nuestra disposición como nunca antes lo había estado. La pregunta aquí es, ¿qué nos afecta? De todo cuanto nos hostiga, ¿qué logra pasar por mí o en mí, dejando una huella, un rastro, una marca?; retomando las palabras de Larrosa (2009, p. 25), "que 'eso que me pasa' al pasar por mí, deja una huella". Sabemos muchas cosas, pero ¿cuánto de aquello que sabemos logra trastocar nuestra práctica educativa y ponernos en cuestión?

En esta línea de pensamiento, podemos referir el trabajo de Walter Benjamin (1987) en su texto "Experiencia y pobreza", en el cual el autor nos lleva a reflexionar sobre la abundancia de estímulos y la pobreza de experiencias, al afirmar: “[...] admitámoslo: esta pobreza de experiencia es pobreza, pero lo es no solo de experiencias privadas, sino de experiencias de la humanidad. Es, por tanto, una especie de nueva barbarie [...]" (p. 169), planteamientos que, aunque escritos por el autor en 1933, en una época de crisis tras los importantes cambios ocurridos en Europa, ligados a los avances de la técnica, la vida en las ciudades y la gran guerra, resuenan también en nuestras sociedades contemporáneas.

No se trata únicamente de proponer un pensamiento que permita percibir esa realidad que no había sido concebida frente a nuestra práctica educativa como experiencia, se trata también de considerar si la propia vivencia del compromiso educativo puede ser una experiencia para niños y jóvenes. ¿Será posible un (re)pensar la educación como una experiencia, como un experimentar, vivir, aprender, que no se limite solo a contenidos y conocimientos, sino también que se trate de nosotros mismos?

Se hace necesario, entonces, cuestionarnos, en cuanto a nuestra relación como docentes con el cotidiano, con el mundo, si lo que recibimos de él se queda solo limitado a una simple información, que no llega a la dimensión de experiencia ya que no trasciende en formación o transformación de lo que somos. Esto supone, a su vez, tener que elucidar el papel que como docentes asumimos y que socialmente se nos ha sido designado, tanto en los procesos de transformación social como en el juego institucional de un establecimiento de enseñanzas, aspectos que serán abordados en las reflexiones a seguir. 


\section{E1 rol social de los profesores}

Personalmente he sostenido que el hecho de ver a los profesores como intelectuales nos capacita para empezar a repensar y reformar las tradiciones y condiciones que hasta ahora han impedido que los profesores asuman todo su potencial como académicos y profesionales activos y reflexivos.

GIRoux, 1997, p. 65

Uno de los sectores que ha sido cuestionado socialmente, observado y desconocido en muchas ocasiones es la educación. Ciertamente esto no ocurre solo en Colombia, también en muchas partes del mundo en donde el Estado y la sociedad se preguntan cuáles han de ser las condiciones de formación de quienes tienen en sus manos la tarea de educar a aquellos que se encuentran en tránsito generacional. Al respecto, Giroux (1997) presenta un argumento que resulta pertinente en estos análisis, al explicar que el papel de los profesores no se circunscribe únicamente al desarrollo de sus habilidades para enseñar:

\begin{abstract}
Si creemos que el papel de la enseñanza no puede reducirse al simple adiestramiento en las habilidades prácticas sino que, por el contrario, implica la educación de una clase de intelectuales vital para el desarrollo de una sociedad libre, entonces la categoría de intelectual sirve para relacionar el objetivo de la educación de los profesores, de la instrucción pública y del perfeccionamiento de los docentes con los principios mismos necesarios para desarrollar una ordenación y una sociedad democráticas. (Giroux, 1997, p. 60).
\end{abstract}

En este sentido, y en respuesta a las reformas educativas que se venían produciendo entonces, el citado autor plantea la necesidad de transformar las consideraciones desde las cuales socialmente se reconoce la profesión docente. Desafortunadamente, en el país no ha sido posible, al menos en la educación pública escolar, que el Estado y la sociedad admitan la profesionalización de la docencia de la educación básica y media, pese a los ingentes esfuerzos que hacen tanto organismos oficiales como los mismos profesores de proveer y proveerse una educación de calidad que los capacite para el ejercicio de sus funciones. El profesional de la educación, hay que decirlo, está sometido a realizar una serie de demostraciones para avanzar en su proceso de desarrollo, en las que se percibe desconfianza oficial en su formación y en su saber, mismas exigencias que no se hacen a ninguna otra profesión.
De cualquier modo, tal y como expone Giroux (1997), son los docentes quienes deben avanzar en la consolidación social de su rol y para ello propone la idea del docente reflexivo, no solo de su práctica, sino de las relaciones sociales que se producen en la escuela y de las transformaciones que a partir de allí puedan ser percibidas socialmente. A pesar de las múltiples tareas, la política pública que está rezagada en el tiempo, las condiciones laborales, el exiguo reconocimiento de la profesión, entre otros factores, en muchos centros educativos avanzan procesos de innovación, hay producción académica, se piensa la institución de maneras diferentes, todo ello en procura de aportar condiciones diferenciales que trasciendan el hecho puramente educativo.

Es cierto que, mientras el Estado y las comunidades no reconozcan el papel que los profesores como profesionales de la educación juegan en los procesos de transformación social, no se logrará que estos sean apreciados como intelectuales (Giroux, 1997). En el mismo sentido, en tanto que los docentes no se asuman como tal y se ubiquen en el campo académico como generadores de conocimiento, con un saber específico, en este caso, el de la escuela y la pedagogía, será muy difícil avanzar en otros aspectos. El profesor, en su práctica reflexiva, debe orientarse al conocimiento de la realidad, indagar e indagarse sobre las situaciones problemáticas que encuentra en su cotidianidad, sobre rutas posibles para aportar soluciones y fortalecer las experiencias de aprendizaje que construye con sus estudiantes, produciendo así verdaderas transformaciones en la vida escolar. Estos, de manera simple, son los fundamentos de la investigación, que conducen al profesor a la comprensión de lo que vive y de aquello que lo motiva, integrando también la experiencia en la búsqueda de caminos para repensar su rol como docente/investigador.

\section{Los profesores de la educación básica y media como investigadores}

Mucho se habla de los académicos y de sus dotes investigativas, se les reconocen sus esfuerzos y logros en el campo de la producción intelectual, pero no es el mismo caso para quienes laboran en la educación pública escolar, quizá porque tradicionalmente se ha entendido que su trabajo y su capacitación no dan para tanto. Sin embargo, los tiempos van cambiando y también los maestros comienzan a transformar la historia que hasta el momento ha imperado en torno a la formación.

Con el interés del Gobierno nacional de ser miembro de la Organización para la Cooperación y el Desarrollo Económico (OCDE) y en aras de responder 
a las reformas que promueven organismos multilaterales, las exigencias para el país, especialmente por la calidad educativa y el fortalecimiento de la formación docente, se hacen evidentes, lo que, igualmente, convoca a la unidad de esfuerzos para que la educación vaya cumpliendo con estas solicitudes.

En ese camino, el Ministerio de Educación Nacional (MEN) planteó como prioridad, en el proyecto nacional de calidad de la educación, la formación docente, con miras a cualificar las prácticas pedagógicas, haciendo, así mismo, énfasis en la necesidad de lograr su desarrollo personal y profesional, con el ánimo de "responder a las expectativas, necesidades y demandas de la sociedad y contribuir desde su quehacer al proyecto educativo de Nación" (MEN, 2013, p. 3). En efecto, el Ministerio bosqueja la que será en adelante la política pública que orientará los procesos de capacitación de los profesores, a la vez que señala, en cierta forma, una visión social diferente en torno a la concepción de maestro.

A partir de reuniones con las agremiaciones de docentes, las universidades y los representantes del Gobierno, se asumieron cinco ejes de discusión para desarrollar la propuesta formativa:

Eje 1: Componentes del sistema de formación de docentes; eje 2: Los sujetos de la educación y su lugar en el sistema de formación; eje 3: Pedagogía, didáctica y el proceso de enseñanza-aprendizaje; eje 4: Investigación pedagógica como componente multifocal del sistema y eje 5: Evaluación como práctica permanente. (MEN, 2013, pp. 11-12).

La oferta educativa que se diseñó en este momento para los docentes incluyó especializaciones, maestrías y doctorados, la cual se sostiene hoy con apoyo económico del Estado. De lo anterior resulta que la investigación se admite como relevante en los procesos de formación; en tal sentido, el documento del MEN explica:

Considerando que el rol del educador sea este directivo o docente, está mediado por la pedagogía, entendida como un campo del conocimiento científico que tiene como objeto de estudio la educación, en el caso particular, de sus prácticas profesionales, la investigación adquiere un valor fundamental, desde el cual es posible dar pertinencia y sentido al proceso educativo, en tanto favorece la movilización de pensamiento y en consecuencia la innovación creativa del conocimiento. (2013, p. 104).

Asumidos así, desde la normatividad, los procesos de formación docente deberán posibilitar la reflexión de las prácticas, en las que requieren conocimientos que generen la necesidad de volver sobre lo que se hace, y revisar la experiencia y la actividad que se produce dentro y fuera de la escuela. Se establece, entonces, que la investigación no es ajena a la actividad escolar y su cotidianidad. Para ampliar lo expuesto, Osses (2003) señala, citando a Boggino y Rosekrans (2004), que existen tres tipos de maestros investigadores: técnico, práctico y crítico. El técnico centra su interés en los resultados, por ello trabaja para aumentar la eficacia del proceso educativo; el maestro investigador práctico le apunta, desde una perspectiva interpretativa, a comprender la situación educativa, es autónomo y activo, reflexiona sobre su práctica e introduce cambios, y vincula a otros en sus procesos cuando es requerido; finalmente, el maestro crítico se caracteriza como aquel que fortalece su práctica y, desde la crítica, observa los espacios educativo y social pensando en la transformación de su realidad (Osses, 2003).

Considerando estos presupuestos, en nuestro proyecto educativo institucional, el docente es reconocido como un investigador crítico que afina su mirada a las problemáticas del entorno que pueden llegar a impactar la vida escolar; en este sentido, los estudiantes son también investigadores $\mathrm{y}$, a la vez, generadores de soluciones a las situaciones que quizá ellos mismos estén produciendo.

Gutiérrez (2015) expone, en torno a los estudios de Cabra (2013), que la "investigación y la innovación, se han posicionado como nociones centrales en el discurso sobre la formación profesional de los maestros. A su vez, se han convertido en referentes de calidad y acreditación de los programas" (p. 9). Si bien este es un tema álgido de la política pública, ha requerido igualmente de la participación de las facultades de educación, en el sentido de desarrollar las habilidades de los profesores en formación para que se pueda establecer con formalidad la investigación y la innovación, aspectos considerados fundamentales en los procesos formativos y posteriormente escolares.

La profesión docente debe resignificarse en el entorno educativo a partir de la reflexión de la práctica, la experiencia, la investigación y la innovación, en la comprensión de que solo de este modo será posible mejorar y alcanzar la transformación que se requiere para llegar a los procesos de calidad que se le han encargado. Ortiz y Suárez (2009) insisten en que

La profesionalización requiere de un mecanismo que permita visualizar las múltiples relaciones que se generan en la formación de maestros, esto es: la investigación educativa, la investigación pedagógica y la investigación formativa, el maestro investigador y la profesionalización misma, en un escenario 
adverso para el logro protagónico que se le exige al maestro dentro del cambio educativo. (p. 76).

Desde la perspectiva investigativa, los docentes necesitan intervenir sus procesos actuales de manera crítica, bien sea el currículo, sus relaciones escolares, la relación con la experiencia, el entorno y las familias, todo ello mediante el diálogo, la acción reflexiva (reflexión sobre la práctica-en contexto) y la reflexión en la acción (pensar en medio de la acción-indagar), como ha sido también expuesto por Dewey (Aljure, 2015).

\section{La investigación en la escuela}

Ahora bien, al referirnos de manera concreta a la investigación en la escuela como método de enseñanza, debemos reconocer que es un concepto en el que se ha venido trabajando en muchas partes del mundo; uno de sus propósitos es encausar los procesos escolares hacia el manejo de métodos que fortalezcan la producción de conocimiento con el acompañamiento del profesor. La idea es que los estudiantes se vinculen a la investigación y que, mediante el desarrollo de competencias en este campo, puedan acceder a otras técnicas formativas en las que ellos mismos sean gestores de su aprendizaje, y puedan realizar conexiones entre teoría y práctica.

Con frecuencia, en el ámbito educativo, se debate la forma como se pueden llevar al aula metodologías más dinámicas que movilicen el pensamiento y den cabida al aprendizaje, sin embargo, hay autores que indican que es muy difícil introducir nuevas formas de hacer en el proceso de aula si antes no se superan unas condiciones que se presentan en el ejercicio de la acción educativa. Cortés (2003) comenta al respecto:

El aula de clase es descrita y vivida por muchos como un espacio jerarquizado y regulado en el que se evidencian estructuras de poder que determinan, por tanto, la dinámica de las relaciones sico-sociales de los allí involucrados: Los procesos pedagógicos que en ella se dan están prescritos por formas particulares de relación, en la que los roles están determinados desde un imaginario que remite a reconocer como válido solo una clase de conocimiento; el académico, solo un tipo de orden; el institucional, solo una forma de escuela; la que no acepta diferencias y un solo un tipo de maestro, el transmisor de conocimientos. (p. 4).

Algunas de estas condiciones han ido en franco mejoramiento, sin que ello quiera decir que se han superado del todo; hay otras visiones del aula, el impacto de la tecnología, la integración de nuevas formas del conocimiento y, en fin, aspectos que han puesto en discusión el uso de nuevas metodologías relacionadas con la interacción con todo tipo de artefactos, que hoy se encuentran al alcance de los estudiantes. Básicamente, es necesario pensar en una seria reforma de la educación desde las políticas públicas, el currículo, entre otras, que renueven y promuevan nuevos estilos de enseñanza.

La investigación, que ya ha entrado en muchas aulas de clase, configurando incluso dinámicas institucionales, como es el caso de la experiencia "Hacia un aprendizaje comprensivo de la investigación”, es entonces reconocida como una posibilidad que no hay que perder de vista en la búsqueda de dotar de sentido el hecho educativo e inspirándonos, como lo referimos al inicio de este texto, en nuestra propia experiencia.

\section{A modo de conclusión}

Tal vez lo que en estas líneas hemos abordado tenga una intención reiterativa de aludir a una mirada de la investigación desde la perspectiva de la experiencia y la realidad, que nos permita una aproximación al mundo y su cotidianidad. Sin embargo, proponemos aquí un distanciamiento con el tono repetido de la palabra experiencia en el ámbito educativo, que hace que su uso se torne, en cierto modo, banalizado, perdiendo la posibilidad de reconocer las múltiples aristas que pueden ser tejidas alrededor de sus enormes posibilidades teóricas, críticas y prácticas.

Aventurarnos a pensar la experiencia educativa como aquello que viabiliza procesos investigativos a partir del ejercicio "Hacia un aprendizaje comprensivo de la investigación" integrado a las dinámicas del Colegio Pablo Neruda es proponer, en primer lugar, abordar lo educativo a partir de lo vivido, de lo que se vive. Pero, principalmente, es aproximarnos a lo que alguien vive, es decir, a lo que personas concretas experimentan en sí mismas, y al decir "alguien" no nos estamos refiriendo solamente a nosotros como investigadores, sino también a nosotros como profesores y estudiantes, e, inclusive, a nosotros como sociedad, gobierno, escuela.

Hablar de la investigación en el contexto escolar del Colegio Pablo Neruda es proponer también un tipo de maestro crítico, reflexivo de su práctica y conocedor de su entorno, que se cuestione y que transforme. Es en estos temas en donde la pedagogía crítica se yergue como el elemento que orienta el proceso de enseñanza-aprendizaje más allá de los programas y de las limitaciones de tiempo y espacio, configurando el escenario escolar como un lugar abierto para la innovación, la acción y para forjar esa escuela que soñamos. 
El profesor, en su práctica reflexiva, debe entonces focalizar su mirada en la investigación desde el lenguaje de la experiencia, es decir, orientarse al conocimiento de la realidad, indagar e indagarse sobre las situaciones problemáticas que encuentra en su cotidianidad, sobre las rutas posibles para aportar soluciones y mejorar las experiencias de aprendizaje que teje con sus estudiantes, produciendo así verdaderas transformaciones en la vida escolar. Estos, de manera básica, son los fundamentos de un ejercicio investigativo que lleva al profesor a comprender lo que vive, lo que lo conmueve y lo que lo motiva, integrando también la experiencia en la búsqueda de caminos posibles para repensar su rol como docente/investigador.

\section{Referencias}

Abraham, T. (2011). El último Foucault. Buenos Aires: Sudamericana.

Aljure, L. (2015). Producción de conocimiento a nivel escolar: el rol del docente investigador. Compartir-Palabra Maestra. Gimnasio Campestre, El Astrolabio. Recuperado de https://goo.gl/1jKkj1

Arendt, H. (2005). Ensayos de comprensión 1930-1954. Escritos no reunidos e inéditos de Hannah Arendt. A. Serrano (Trad.). Madrid: Caparrós.

Arendt, H. (2016). La crisis en la educación. En A. Poljak (Trad.), Entre el pasado y el futuro. Ocho ejercicios sobre reflexión política (p. 9). Barcelona: Península.

Arnal, J., Del Rincón, D. y La Torre, A. (1994). Investigación educativa: fundamentos y metodología. Barcelona: Lapor.

Benjamin, W. (1987 [1933]). Experiencia y pobreza. En W. Benjamin, Discursos interrumpidos I. Filosofía del arte y de la historia (p. 168). Madrid: Taurus.

Breton, A. (2009). Primer manifiesto del surrealismo (1924). En A. González, F. Calvo y S. Marchán (Eds.), Escritos de arte de Vanguardia 1900/1945 (pp. 392409). Madrid: Akal.

Boggino, N. y Rosekrans, K. (2004). Investigación-acción: reflexión crítica sobre la práctica educativa. Buenos Aires: Homo Sapiens.
Cabra-Torres, F. (2013). La investigación e innovación en la formación inicial de docentes. Aportes para la reflexión y el debate. Bogotá: Pontificia Universidad Javeriana.

Cortés, R. (2003). La investigación en el aula y la innovación pedagógica. Bogotá: Instituto para la Investigación Educativa y el Desarrollo Pedagógico (IDEP).

Freire, P. (2006). Pedagogía de la autonomía. Saberes necesarios para la práctica educativa. México D.F:: Siglo XXI.

Giroux, H. (1997). Los profesores como intelectuales: hacia una pedagogía crítica del aprendizaje. Barcelona: Paidós Ibérica.

Gutiérrez, M. (2015). ¿Maestros investigadores e innovadores? ¿Cuál es el panorama de la formación para la innovación y la investigación en los programas de licenciatura del país? Pesquisa Ciencias Sociales/Educación. Recuperado de https://goo.gl/E7X8b5

Kohan, W. (2015). Viajar para vivir: ensayar. La vida como escuela de viaje. Buenos Aires: Miño y Dávila.

Larrosa, J. (2009). Experiencia y alteridad en educación. En J. Larrosa y C. Skliar (Eds.), Experiencia y alteridad en educación (pp. 13-44). Buenos Aires: Homo Sapiens/ Flacso.

Larrosa, J. y Kohan, W. (2014). Apresentação da coleção. En J. Masschelein y M. Simons (Eds.), A pedagogia, a democracia, a escola (pp. 4-5). Belo Horizonte: Autêntica.

Masschelein, J. y Simons, M. (2014). Defensa de la escuela. Una cuestión pública. Buenos Aires: Miño y Dávila.

Ministerio de Educación Nacional (MEN), Viceministerio de Educación Preescolar, Básica y Media (2013). Sistema colombiano de formación de educadores y lineamientos de política. Bogotá: Imprenta Nacional de Colombia.

Osses, S. (2003). Hacia la autonomía profesional del docente. El profesor como investigador en el aula. Proyecto Fondecyt Regular 1030278. Recuperado de https://goo.gl/oNhj2f

Ortiz, R. y Suárez, J. (2009). La formación de maestros y la noción maestro investigador (1996-2005) un espacio para la reflexión y el debate (tesis inédita de maestría). Universidad de Antioquia, Medellín, Colombia.

Roso, M. (2010). Investigación en enseñanza secundaria: los “jóvenes investigadores". Tejuelo, 9. Recuperado de https://goo.gl/UnGLNN-100-120 
to

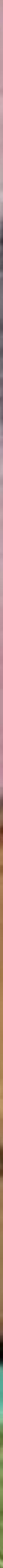

